\title{
Impact of vagal nerve stimulation on quality of life in drug-resistant epilepsy
}

\author{
Nicoleta Tohanean, Cristina Pinzaru, Luminita Mirea, Lacramioara Perju-Dumbrava \\ Neuroscience Department, "Iuliu Hatieganu" University of Medicine and Pharmacy, \\ Cluj-Napoca, Romania
}

\begin{abstract}
Background and objective. Vagal nerve stimulation (VNS) represents an alternative therapy for intractable epilepsy. The aim of this study is to analyse seizure reduction and the life quality of these patients.

Material and methods. We prospectively examined 28 adult patients treated with VNS that were followed-up at least 6 months after the surgery and we recorded the number of seizures and any other changes. 16 epilepsy patients completed the Quality of Life in Epilepsy-31 questionnaire (QOLIE-31).

Results. Our data revealed that $64 \%$ of patients were responders with more than $50 \%$ seizure reduction. According to McHugh classification of seizure freedom, 36\% patients are in class I ( $80-100 \%$ seizure reduction), $29 \%$ class II (50-79\% reduction), $21 \%$ class III (less than $50 \%$ reduction), $7 \%$ class IV (magnet benefit only) and $7 \%$ class $\mathrm{V}$ (no improvement). Only 8 patients presented mild adverse effects, such as hoarseness, fatigue and cough. Life quality has improved for $68 \%$ patients. There is a strong correlation between life quality and health and a mild positive relation with the seizure reduction.

Conclusions. VNS improves life quality for more than half of patients and is a therapy to consider in refractory epilepsy.
\end{abstract}

Keywords: vagal nerve stimulation, epilepsy, life quality

\section{INTRODUCTION}

The failure in keeping seizures under control can lead to a significant decrease in life quality of epileptic patients. Up to one third of patients who suffer from the disease have intractable epilepsy. The International League Against Epilepsy (ILAE) defines epilepsy as refractory in case of inability to stop the seizures with at least two complete medical schemes (1).

Vagal nerve stimulation (VNS) is an alternative for medical or surgical refractory epilepsy and it is used in the case of failure or imposibility in performing an antiepileptic surgery, in the case of not obtaining clinical results after at least two medication plans, or in the case of medication intollerance of any kind. It has been used both for partial or generalized seizures, children or adults, for more than 30 years (2).

The device is similar to a cardiac pacemaker and it is attached subcutaneously in the left subclavicu- lar area, while the electrods are placed in the left vagus nerve. Although arrhythmias ar extremely rare, it is connected only the the left vagal nerve to even diminish that risk. The generator delivers intermittent electrical signals of low frequency to the nerve pathways (3). After the surgery, some parameters are noninvasively adjusted, including the intensity (output current), frequency, on time and off time. Patients also receive a magnet which can suspend the electrical stimulation while it is held in the generator area, thus interupting the seizure at its beginning (5).

The mechanism of action is not completely elucidated because of the difficulty in animal studies, but it is based on the change in neurotransimitters such as serotonin, noradrenalin and GABA. The tenth cranial nerve has wide projections over some structures of the brain that are important in epileptogenesis, such as thalamus, amygdala, raphe nucleus, locus coeruleus. The electrical impulses mi- 
ght transform synchronous cortical activity to desynchronous and reduce the cortical excitability (4).

Due to the complex pathophysiology, VNS is indicated as an alternative to several diseases in case of medical failure. It is a possible therapy for medical refractory epilepsy, antiepileptic drug severe adverse effects, the impossibility in performing a curative antiepileptic surgery, uncontrolled status epilepticus, chronic headache, neurodegenerative disease, schizophrenia, autism, medical refractory depression (6-8).

Early complications such as bradycardia, asystole, hematoma, infections, vocal cord paralysis are related to the surgery technique, while the most frequent late complications include hoarseness, dyspnoea and coughing (9).

In an evidence-based guideline which evaluated 1274 studies, it was concluded that $55 \%$ of patients had at least a 50\% seizure reduction with improvement over time, with the best response for generalized seizure type. The anti-depressive effect is an additional benefice. Changes in stimulation parameters are not completely accepted by all authors to increase the effect. The efficacy of the magnet varies according to different studies from 25 to $75 \%$ (10). Another meta-analysis finds that only $30 \%$ had a 50\% seizure reduction (11), but all literature data agrees that VNS has a significant lower hospitalisation rate and it has a benefice on epileptic patients.

\section{MATHERIAL AND METODS}

Our study included 28 adult patients from Cluj-Napoca Neurology Clinic with the medium age of 32 years old, which were regularly followed-up after the VNS implantation. We recorded the clinical parameters of the patients, the number of seizures and adverse effects for a medium period of 20 months.

The inclusion criteria were the presence of vagal nerve stimulation therapy for epilepsy in adult patients. We excluded all children and the patients who withdrew from the study or deceased from other causes.

For 16 patients we evaluated the Quality of Life in Epilepsy-31 questionnaire (QOLIE-31) consisting of personal ratings of overall life quality, emo- tional well-being, seizure worry, energy, cognitive function, medication effects and social life. For each category we calculated the final score which reflects the life quality on that specific field, and also the total score. Based on a group of 304 epilepsy patients, it was developed a table with pairing between any specific or final score and a T-score. $\mathrm{T}$-scores are linear transformations of the scores with the mean of 50 and a standard deviation of 10 , representing that a $\mathrm{T}$-score of 50 is correlated with the mean of the standard cohort (12).

TABLE 1. Clinical characteristics of the VNS patients

\begin{tabular}{|c|c|}
\hline Characteristics & $\begin{array}{l}\text { Total number of patients } \\
\qquad(n=28)\end{array}$ \\
\hline Sex males & $15(54 \%)$ \\
\hline females & $13(46 \%)$ \\
\hline Age at VNS implanta on (years) & $32,9 \pm 9,7(18-52)$ \\
\hline Age at epilepsy onset (years) & $12,5 \pm 7,5(0,25-27)$ \\
\hline Epilepsy dura on (years) & $20,7 \pm 11,1(3-48)$ \\
\hline Follow-up me (months) & $20,6 \pm 9,1(6-35)$ \\
\hline Mental retarda on present & $12(43 \%)$ \\
\hline absent & $16(57 \%)$ \\
\hline Number of an epilep c drugs & $2,6 \pm 0,8$ \\
\hline
\end{tabular}

Values are expressed in mean \pm standard devia on (range) or $\mathrm{n}(\%)$.

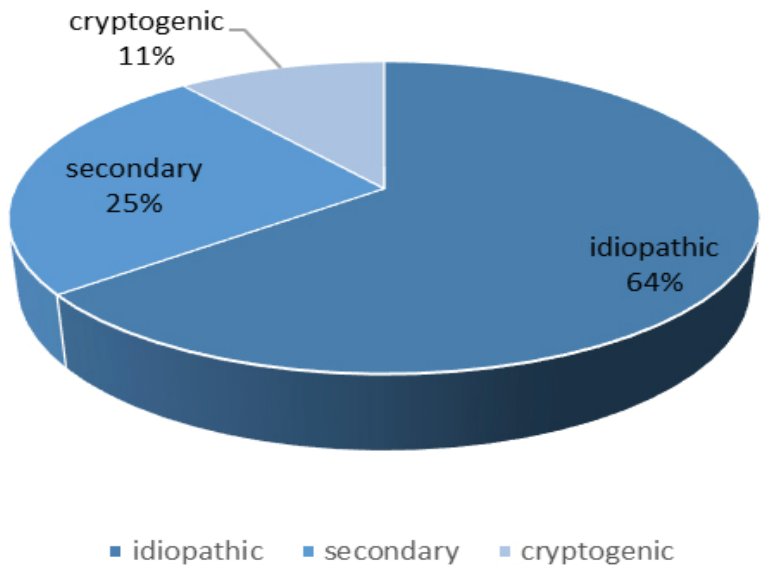

FIGURE 1. Epilepsy type in VNS patients

\section{RESULTS}

\section{Seizure frequency}

For the patients in our study, the seizure frequency is extremely varied from one to 150 episodes per month, as it is presented in Fig. 2. At least 6 months after VNS implantation, we can observe in Fig. 3 a different distribution of frequencies with a general lower severity. 


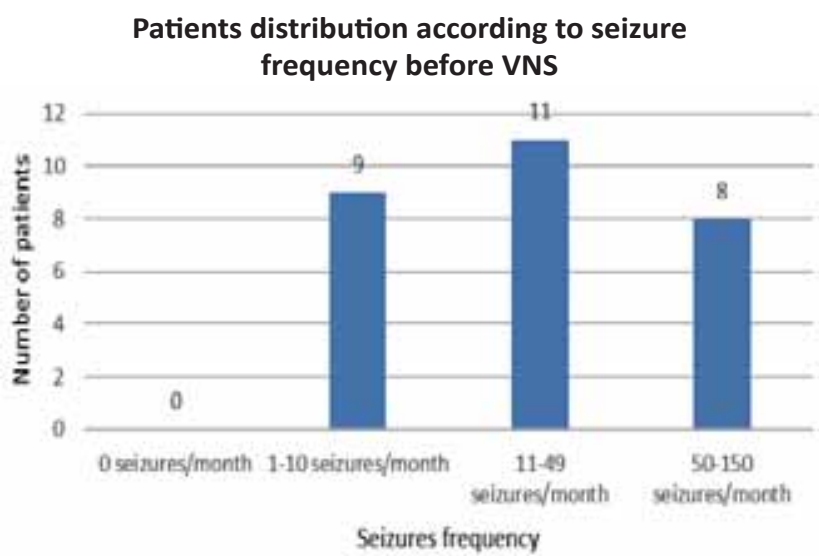

FIGURE 2. Seizure frequency before VNS

Patients distribution according to seziure frequency after VNS

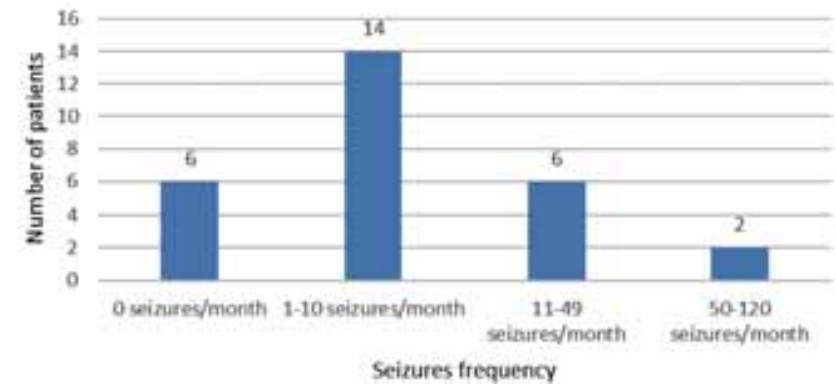

FIGURE 3. Seizure frequency after VNS

\section{Seizure frequency reduction}

For each patient we calculated the reduction rate according to the declared frequency anterior and after VNS implantation and we analysed the data using the McHugh and modified Engel classifications.

After a medium period of 20 months, the majority of patients presented important improvements in epileptic episodes. According to McHugh classification from Fig. 4, 10 patients were in class I with a reduction rate of more than $80 \%, 8$ patients were included in class II with a reduction of $50-79 \%, 6$ were in class III with less than $50 \%$ reduction, 2 presented no improvement and 2 had some benefic effects of the magnet.

In modified Engel classification from Fig. 5, complete seizure freedom was declared by 6 patients, one presented with more than $90 \%$ reduction, 11 with $50-90 \%$ reduction and 1- patients had less than $49 \%$ reduction in seizure frequency.

Regarding the gender, our evaluation revealed no differentiation between females and males in the efficacy of the VNS therapy. The type of epilepsy might be a predictor of the outcome, idiopathic etiology remaining with the best response.

\section{McHugh classification}

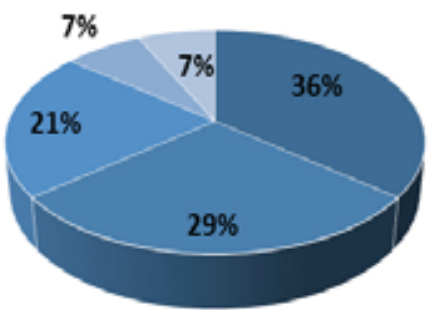

- I (seizure reduction rate 80-100\%) - II (seizure reduction rate 50-79\%)

- III (seizure reduction rate-s0\%) - IV (magnet benefit only)

- $V$ (no improvement)

FIGURE 4. McHugh classification of outcome after VNS implantation

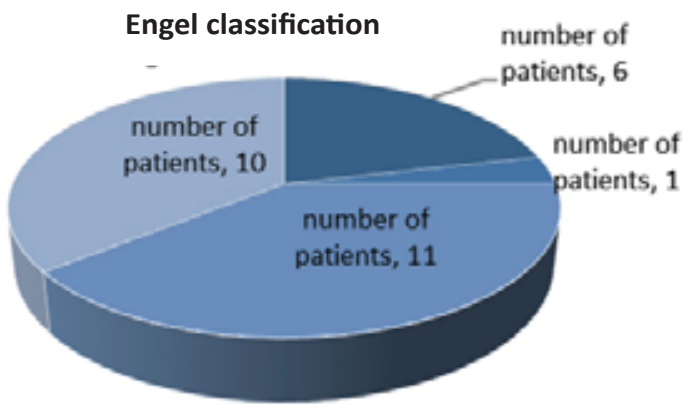

- class I (0 or rare partial seizures)

- class II (>90\% seizure reduction rate or rare complex seizures)

- class III (50-90\% seizure reduction)

" class IV (<50\% seizure reduction)

FIGURE 5. Modified Engel classification of outcome after VNS implantation

\section{The overall response to VNS}

We classified all 28 patients in responders(with a reduction in seizure frequency of more thena $50 \%$ ) and non responders ( $<50 \%$ reduction). $64 \%$ of patients responded to this form of treatment and $36 \%$ did not declared a significant efficacy (Fig. 6).

\section{Responsiveness distribution}

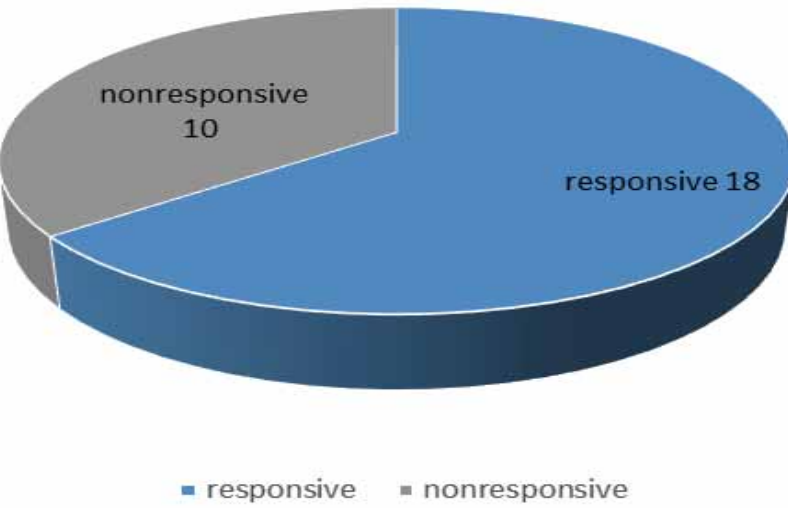

FIGURE 6. Responsiveness distribution 


\section{Adverse effects}

A majority of 20 patients had only temporary adverse effects, but 6 patients had a persistent dysphonia, one presented cough and one patients had fatique.

\section{Overall life quality and health}

The 16 patients from the study who completed the questionnaire accorded a grade from 1 to 10 for the overall life quality they consider to be characteristic most of the time.

To analyse the correlation between the overall life quality and seizure frequency reduction, we used Pearson correlation (Fig. 7). With an r of 0,39 there is a mild positive correlation between the two variables.

The way patients consider their overall health is very strong related to their life quality. In a Pearson correlation between overall life quality grade and the health grade (Fig. 8), we find a strong positive linear relation with a $\mathrm{r}=0,76$.

\section{QOLIE-31 scores}

We calculated the T-scores for all categories, a score equal to 50 reflecting the mean of the responses from the standard epilepsy cohort. The results were very closed to the 50 value, in general lower. The mean from all T-scores from worries about a new seizure was 43,1 , overall life quality had a T-scores mean of 46,1, emotions 46, energy 48,9, cognitive function 49,8, medication adverse effects 51,3 , social implications were the most affected with a mean of 40 .

We evaluated a possible correlation between the final T-score and seizure reduction rate using Pearson correlation (Fig. 9). With a result of 0,13 , there is not any significant correlation between seizure reduction and the score which represents the life quality considered by the patient.

\section{Life quality changes}

We asked the 16 patients in the questionnaire to evaluate how VNS therapy has changed their lives,

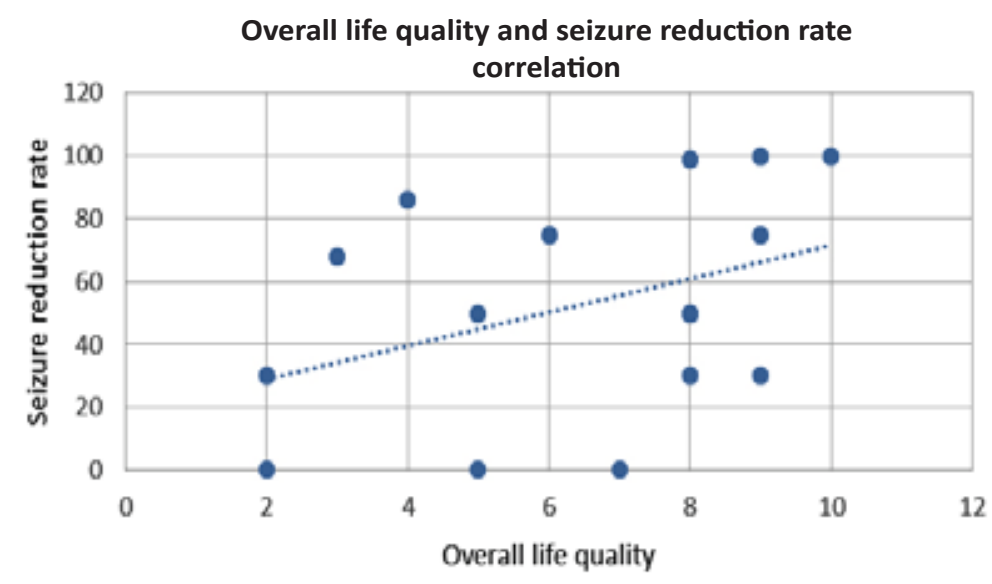

FIGURE 7. The Pearson correlation between overall life quality grade and seizure reduction rate

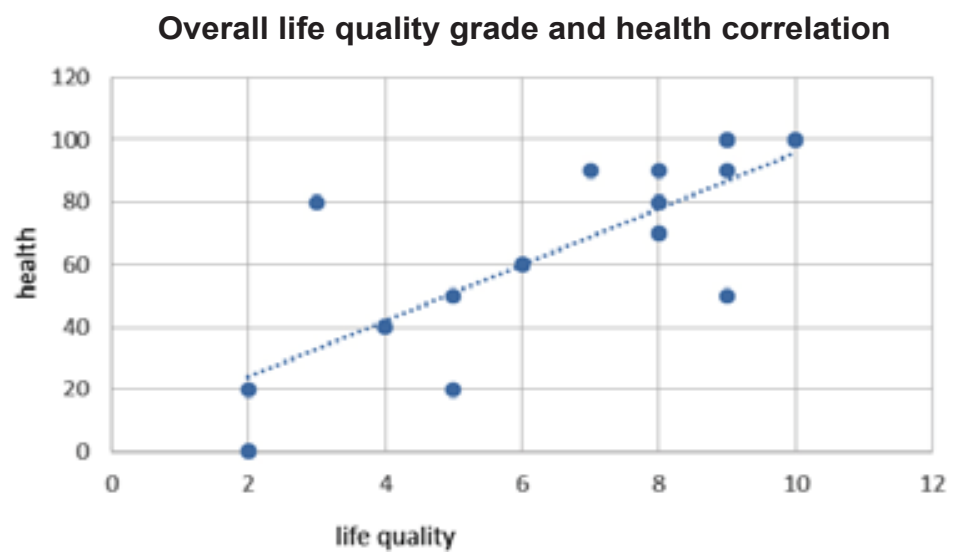

FIGURE 8. The Pearson correlation between overall life quality and health grades 


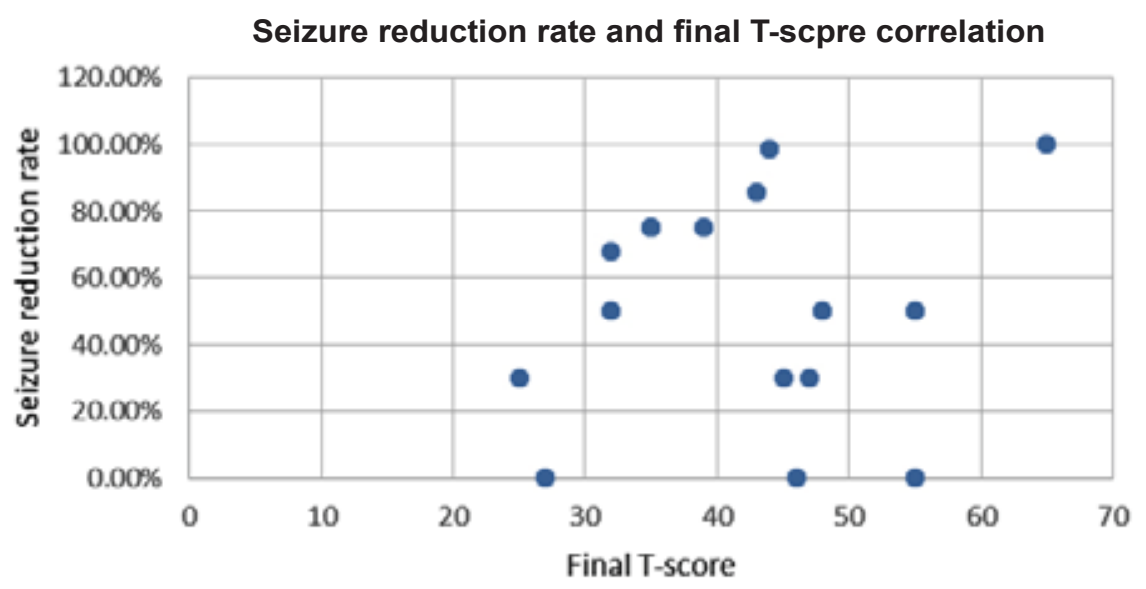

FIGURE 9. The Pearson correlation between seizure reduction rate and final T-score

by choosing one of the predefined answers. $10 \mathrm{pa}-$ tients declared a moderate improvement, one considered a total life changing treatment, one has been a little influenced and 4 noticed no difference. From these patients, 11 consider a favourable treatment.

\section{DISCUSSION}

Vagal nerve stimulation is an accepted alternative treatment for refractory epilepsy. It has a documented role in reducing seizure frequency and intensity in time and interrupting the seizure at its beginning.

A large number of literature studies are focused on the reduction rate and its efficacy, but only a few evaluate the impact of VNS therapy on life quality. The contribution of our study consists in analysing a therapeutic method not as well known and which, although in use for many years in other countries, it is still just at its beginning in our region. Also, studying QOLIE-31 on patients with vagal nerve stimulation represents a less common approach.

The limitations of the study are based on the small number of subjects, due to the extremely rare utilisation of VNS therapy in the region. Other errors might occur in collecting data from patients' declarations and from their families as well as accessing medical files.

\section{CONCLUSIONS}

Our data show that $64 \%$ of total 28 patients had more than $50 \%$ response in seizure reduction and the adverse effects were minor.

The quality of life scores in QOLIE-31 applied to the 16 patients were similar to the mean value of epileptic patients, with a lower tendency. The data show no correlation between the QOLIE-31 final score and the seizure reduction rate, which suggests that vagal nerve stimulation is a possible therapy for reducing the number of seizures, but it is not necessarily related to the patient's perception about their life.

Finally, vagal nerve stimulation is an effective option for medical and surgical resistant epilepsy.

Conflict of interest: none declared Financial support: none declared

\section{REFERENCES}

1. Kwan P., Arzimanoglou A., Berg A.T., Brodie M.J., Hausen W.A., Mathern G. et al. Definition of drug resistant epilepsy.Consensus proposal by the ad hoc Task Force of the ILAE Commision on Therapeutic Strategies. Epilepsia.

2. Vonck K., Boon P. The mechanism of action of vagus nerve stimulation therapy. Reference Centre for Refractory Epilepsy. Ghent. 2008. p. $97-100$

3. DeGiorgio C.M., Krahl S.E. Neurostimulation for drug-resistant epilepsy. Continuum. 2013; 19(3): 743-55.

4. Nemeroff C., Mayberg H., Krahl S., McNamara J., Frazer A., Henry T. et II. VNS Therapy in Treatment-Resistant Depression:

Clinical Evidence and Putative Neurobiological Mechanisms. Neuropsychopharmacology. 2006; 31:1345-55.

5. Yamamoto Takamichi. Vagus nerve stimulation therapy: indications, programing, and outcomes. 2015; 55:407-15.

6. Cervenka M.C. Nonpharmacologic Treatments of Status Epilepticus. In: Drislane F, Kaplan MBBS P, editors. Status Epilepticus. Current Clinical Neurology. Springer, 2017 Nov; pp 215-223.

7. Garcia-Oscos F., Pena D., Housini M., Cheng D., Lopez D., Borland M. Vagal nerve stimulation blocks interleukin 6-dependent synaptic hyperexcitability induced by lipopolysaccharide-induced 
acute stress in the rodent prefrontal cortex. Brain, Behavior, and Immunity. 2014; 43:149-58.

8. Cristancho M., Cristancho P., Altinay M., O'Reardon J. Vagus nerve stimulation (VNS). In: Camprodon J., Rauch S., Greenberg B., Dougherty D., editors. Psychiatric Neurotherapeutics. Current Clinical Psychiatry. New York:Humana Press; 2016.p.99-116.

9. Giordano F., Zicca A., Barba C., Guerrini R., Genitori L. Vagus nerve stimulation: Surgical technique of implantation and revision and related morbidity. Epilepsia. 2017; 58: 85-90.
10. Morris G., Gloss D., Buchhalter J., Mack K.J,. Nickels K., Harden C. Evidence-based guideline update: Vagus nerve stimulation for the treatment of epilepsy. American Academy of Neurology. 2013; 1453-8.

11. Chambers A., Bowen J.M. Electrical stimulation for drug-resistant epilepsy: an evidence-based analysis. Ontario Health Technology Assessment Series. 2013; 13(18): 1-37.

12. Vickrey B., Perrine K., Hays R., Hermann B., Cramer J., Meador K. et all. Quality of life in epilepsy QOLIE-31 Soring manual. $1^{\text {st }}$ edition. Rand.1993. 